\title{
Load Balancing in Structured P2P Systems using Server Reassignment Technique
}

\author{
S.S.Patil \\ Rajarambapu Institute of Technology Rajaramnagar/CSE \\ Sangli, India \\ S.K.Shirgave \\ Dattajirao Kadam College of Textile Engineering Inchalkaranji/CSE \\ Sangli, India
}

\begin{abstract}
Load balancing among application layer peer-to-peer (P2P) networks is critical for its effectiveness but, is considered to be the most important development for next-generation internet infrastructure. Most structured P2P systems rely on ID-space partitioning schemes to solve the load imbalance problem and have been known to result in an imbalance factor of $\theta(\log \mathrm{N})$ in the zone sizes.

Two important contributions to minimize the same are proposed in [1]. First, the virtual-server-based load balancing problem using an optimization-based approach and deriving proposal in general and its advantages over previous strategies. Second, characterizing the effect of heterogeneity on load balancing algorithm performance and the conditions in which heterogeneity may be easy or hard to deal with based on an extensive study of a wide spectrum of load and capacity scenarios.
\end{abstract}

The full text of the article is not available in the cache. Kindly refer the IJCA digital library at www.ijcaonline.org for the complete article. In case, you face problems while downloading the full-text, please send a mail to editor at editor@ijcaonline.org 